\title{
ОСОБЕННОСТИ СТРУКТУРНО-ФАЗОВЫХ ПРЕВРАЩЕНИЙ ПРИ ОТЖИГЕ ПРОТОНООБМЕННЫХ СЛОЕВ НА Z-СРЕЗЕ КРИСТАЛЛА НИОБАТА ЛИТИЯ
}

\author{
() 2018 И. В. Петухов, С. С. Мушинский, М. А. Пермякова, В. И. Кичигин, \\ Л. Н. Малинина, А. Б. Волынцев \\ Пермский государственный национальный исследовательский университет, \\ ул. Букирева, 15, 614990 Пермь, Россия \\ e-mail: Petukhov-309@yandex.ru
}

Поступила в редакцию 17.05.2018

\begin{abstract}
Аннотация. С использованием методов оптической микроскопии в поляризованном свете, модовой спектроскопии, ИК-спектроскопии и рентгеноструктурного анализа подтверждена последовательность фазовых превращений, происходящих при отжиге протонообменных слоев на Z-срезе кристалла ниобата лития: $\beta_{2}-, \beta_{1}$-фазы $\rightarrow \kappa_{2}$-фаза $\rightarrow \kappa_{1}$-фаза $\rightarrow \alpha$-фаза. Установлено, что в процессе отжига в ходе фазовых превращений когерентность слоев, образованных из различных фаз, не нарушается, что обусловлено меньшей величиной деформаций (напряжений) формирующихся протонообменных фаз по сравнению с деформациями (напряжениями) на Х-срезе кристалла.
\end{abstract}

Ключевые слова: ниобат лития, Z-срез, протонный обмен, отжиг, фазовые превращения.

DOI: https://doi.org/10.17308/kcmf.2018.20/581

\section{ВВЕДЕНИЕ}

Кристаллы ниобата лития широко применяются в интегральной оптике для изготовления интегрально-оптических фазовых модуляторов [1]. Распространенным способом формирования оптических волноводов в поверхностных слоях кристалла $\mathrm{LiNbO}_{3}$ является протонный обмен (ПО) с последующим отжигом $[1,2]$. Оптические и электрооптические свойства ПО-волноводов зависят от их фазового состава $[2,3]$. В зависимости от условий протонного обмена и отжига возможно образование до 7 протонообменных фаз твердого раствора $\mathrm{H}_{\mathrm{x}} \mathrm{Li}_{1-\mathrm{x}} \mathrm{NbO}_{3}$ [2]. Структурно-фазовые диаграммы системы $\mathrm{H}_{\mathrm{x}} \mathrm{Li}_{1-\mathrm{x}} \mathrm{NbO}_{3}$ для $\mathrm{Z}$ - и $\mathrm{X}$-срезов $\mathrm{LiNbO}_{3}$ различаются между собой [2], что необходимо учитывать при формировании ПО-волноводов на различных гранях монокристаллов ниобата лития. Эти различия, в частности, выражаются в том, что ПО-слои, сформированные на Z-срезе, характеризуются меньшими значениями напряжений и деформаций.

Большое значение имеет последовательность и кинетика фазовых превращений, происходящих при отжиге ПО-слоев, поскольку отжиг является одной из основных технологических операций при изготовлении стабильных ПО-волноводов с хорошими электрооптическими свойствами и низкими оптическими потерями $[1,4]$. Имеются расхождения в представлении хода фазовых превращений при отжиге [5-7]. Поэтому необходимы дальнейшие исследования кинетики фазовых превращений в $\mathrm{H}: \mathrm{LiNbO}_{3}$ волноводах при отжиге, особенно на завершающих стадиях перехода в $\alpha$-фазу.

В работах $[8,9]$ с использованием оптической микроскопии, рентгеноструктурного анализа, модовой и ИК-спектроскопии была изучена последовательность и кинетика фазовых превращений при отжиге протонообменных слоев на X-срезе кристалла ниобата лития. Цель данной работы - изучить процессы, происходящие при отжиге протонообменных слоев на Z-срезе, и сопоставить их со структурно-фазовыми превращениями в протонообменных слоях на X-срезе кристалла ниобата лития.

\section{ЭКСПЕРИМЕНТАЛЬНАЯ ЧАСТЬ}

Для проведения исследований использовался Z-срез кристалла ниобата лития конгруэнтного со- 
става производства Sipat. Рабочими образцами служили пластины толщиной 1 мм размером $15 \times 10$ мм, вырезанные из вейфера диаметром 76 мм.

Протонный обмен проводился в закрытом циркониевом реакторе в расплаве бензойной кислоты (ч.д.а.) при температуре $190{ }^{\circ} \mathrm{C}$ в течение 2 ч. Увеличение температуры по сравнению с ПО на Х-срезе $[8,9]$ связано с тем, что диффузия протонов на Z-срезе при протонном обмене протекает более медленно. Поэтому для получения протонообменных слоев примерно одинаковой толщины на различных гранях кристалла ниобата лития температура протонного обмена на Z-срезе повышена. Отжиг образцов проводился последовательно с шагом 1 ч при температуре $330{ }^{\circ} \mathrm{C}$. Суммарная продолжительность отжига достигала 18 ч.

Для исследуемых планарных волноводов определяли профили приращения показателя преломления необыкновенного луча $\Delta n_{\mathrm{e}}(\mathrm{x})$ и значения $\Delta n_{\mathrm{e}}(0)$ на поверхности волноводного слоя. Для определения $\Delta n_{\mathrm{e}}(x)$ и $\Delta n_{\mathrm{e}}(0)$ на планарных волноводах использовали метод модовой спектроскопии. С помощью призменного ввода измеряли эффективные показатели преломления волноводных мод на длине волны $\lambda=0.633$ мкм. Профиль $\Delta n_{\mathrm{e}}(x)$ по глубине волноводного слоя восстанавливался с помощью обратного метода Вентцеля-КрамерсаБриллюэна [10].

Для визуализации структуры ПО-слоев использовали метод оптической микроскопии (Olympus BX 61) с применением методики светлого и темного полей в поляризованном свете.

Рентгенографические исследования образцов ниобата лития проводились на рентгеновском двухкристальном дифрактометре ДРОН-УМ1 в излучении кобальтового анода с использованием длины волны $\lambda_{\beta}=1.62073 \AA$. Регистрировали $\theta / 2 \theta$ кривые. Величина деформации $\varepsilon_{33}$ в направлении нормали к поверхности определялась непосредственно из $\theta / 2 \theta$ кривых измерением углового расстояния $\Delta \theta$ между положением максимума дифракционного отражения от подложки ниобата лития и максимумом дифракционного отражения от соответствующей фазы $\mathrm{H}_{\mathrm{x}} \mathrm{Li}_{1-\mathrm{x}} \mathrm{NbO}_{3}$ в соответствии с формулой Вульфа-Брэгга в дифференциальной форме:

$$
\varepsilon_{33}=-\Delta \theta \operatorname{ctg} \theta,
$$

где $\theta$ - Брэгговский угол отражения, ассоциированный с рассматриваемым семейством кристаллических плоскостей.

Для количественной оценки процессов, происходящих при отжиге, производилась декомпо- зиция дифракционного максимума на несколько пиков, соответствующих тем или иным фазам, образующимся при отжиге. Для декомпозиции использовалась функция Гаусса и метод аппроксимации Левенберга-Марквардта. В ходе расчетов проводилось 100 последовательных итераций для более точного соответствия экспериментальных и расчетных кривых. После декомпозиции пика определяли положение максимумов и интегральную интенсивность линий (площадь пика).

Образцы ниобата лития с планарными ПО-волноводами также исследовали методом ИК-спектроскопии с помощью спектрофотометра Bruker Spectrum Two в частотном диапазоне $400-6000 \mathrm{~cm}^{-1}$ с разрешением $<1 \mathrm{~cm}^{-1}$.

\section{РЕЗУЛЬТАТЫ И ОБСУЖДЕНИЕ}

В результате протонного обмена формируется протонообменный слой, состоящий из $\beta_{1}$ - и $\beta_{2}$-фаз. На это указывает достаточно широкий пик на дифрактограмме (рис. 1a) с максимумом, соответствующим деформации $\varepsilon_{33}=4 \cdot 1 \cdot 10^{-3}$. Данный пик, вероятно, образуется из-за перекрывания пиков $\beta_{1}$ - и $\beta_{2}$-фаз, которым на структурно-фазовой диаграмме соответствуют деформации $\varepsilon_{33}=(3.1 \div 4.2) \cdot 10^{-3}$ и $(4.3 \div 5.7) \cdot 10^{-3}[2]$.

Результаты модовой спектроскопии (рис. 2a) также указывают на образование слоя, состоящего из $\beta_{1}$ - и $\beta_{2}$-фаз, в ходе протонного обмена, что следует из сопоставления значения $\Delta n_{\mathrm{e}}$ на поверхности волновода со значениями на структурно-фазовой диаграмме [2]. Далее в процессе отжига происходит монотонное снижение величины $\Delta n_{\mathrm{e}}(0)$ и увеличение глубины волноводного слоя (рис. $2 b$ ).

Отжиг протонообменного слоя в течение 1 ч приводит к исчезновению пика, соответствующего $\beta_{1}$ - и $\beta_{2}$-фазам, и появлению пика со значением $\varepsilon_{33}=2 \cdot 2 \cdot 10^{-3}$, соответствующего низкотемпературной $\kappa_{2}$-фазе. Данный пик отчетливо различим на дифрактограммах линий (006) и (0012), соответствующих первому и второму порядкам отражения (рис. $1 b)$. Заметной трансформации подвергается и основной пик; он становится асимметричным.

По результатам исследования методом ИК-спектроскопии, в отличие от X-среза, вместо размытого пика поглощения, соответствующего межузельным протонам в $\beta_{2}$-фазе, регистрируется некоторое увеличение интенсивности в области значений $v=3000-3400 \mathrm{~cm}^{-1}$ (рис. 3a). Поскольку протоны $\beta_{2}$-фазы в кристаллической решетке ниобата лития занимают случайные положения, их энергия связи в кристаллической решетке варьируется в 


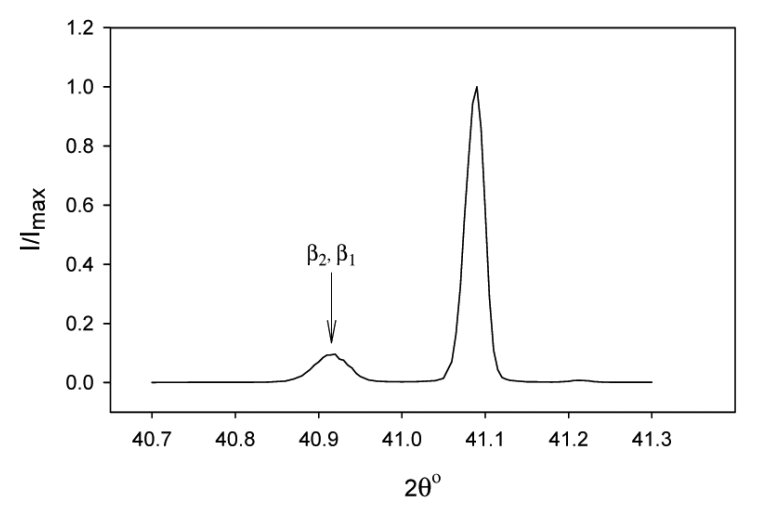

$a$

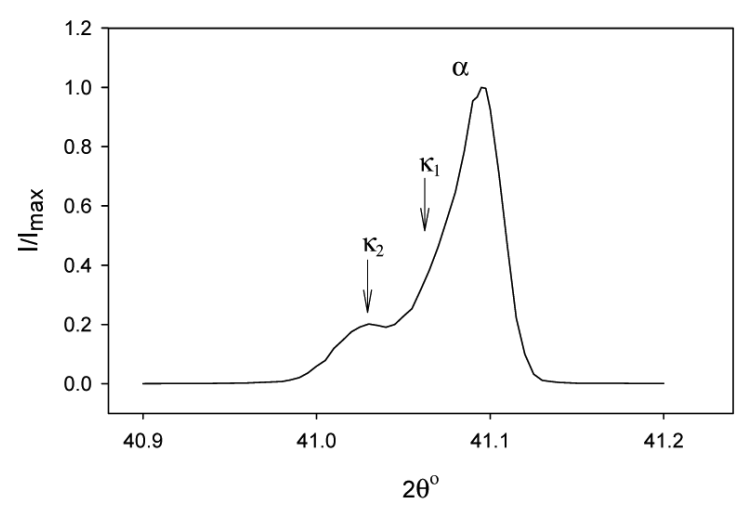

C

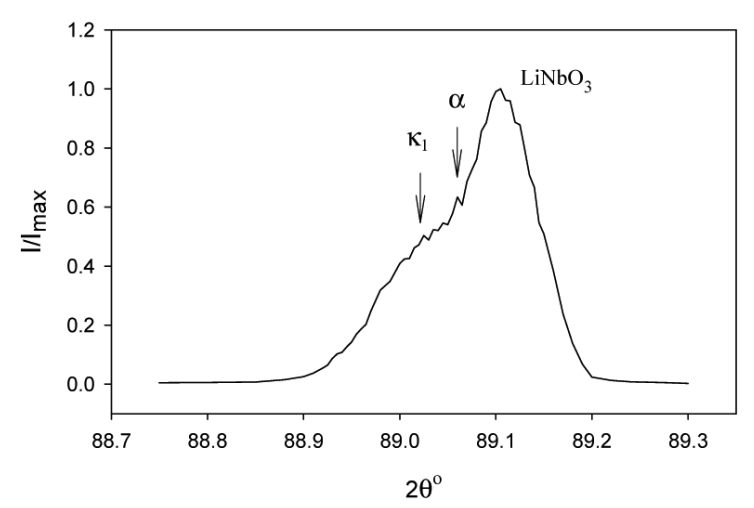

$e$

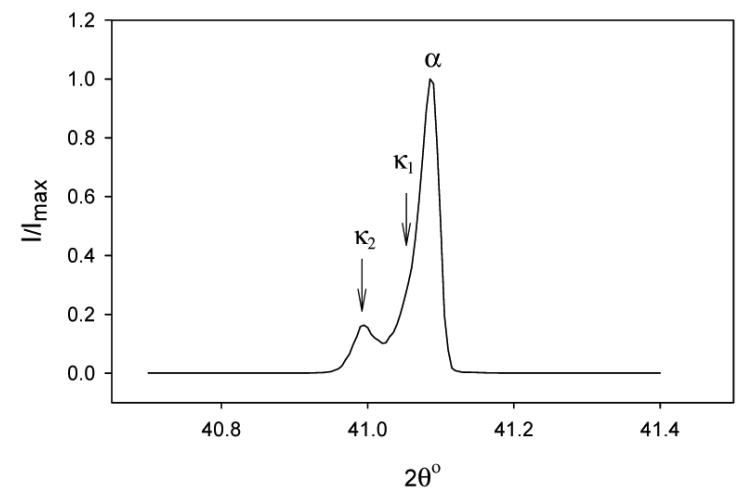

$b$
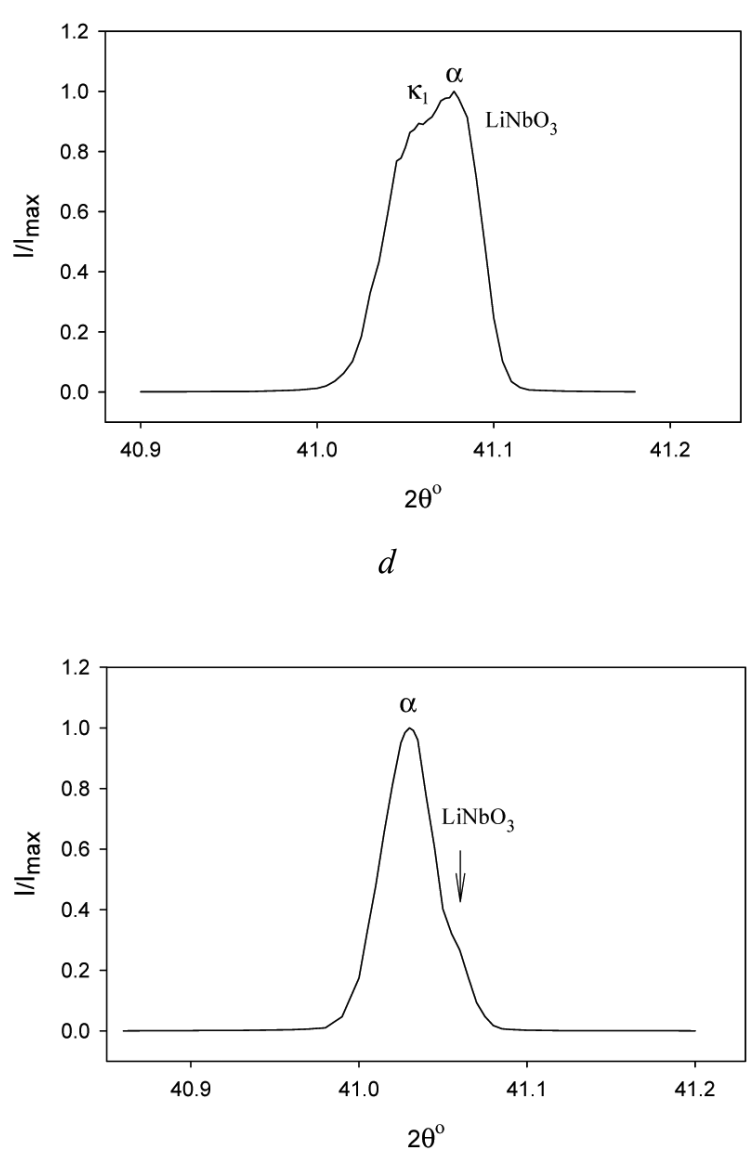

$f$

Рис. 1. $\theta / 2 \theta$ кривые протонированных слоев на кристалле ниобата лития после отжига продолжительностью, ч: $a-0 ; b-1 ; c-2 ; d, e-6 ; f-15 ; a-d, f$ - отражение (006); $e$ - отражение (0012)

[Fig. 1. $\theta / 2 \theta$ curves for proton-exchanged layers in lithium niobate crystal after annealing of duration, h: $a-0 ; b-1 ; c$ $-2 ; d, e-6 ; f-15 ; a-d, f-(006)$ reflection; $e-(0012)$ reflection] 

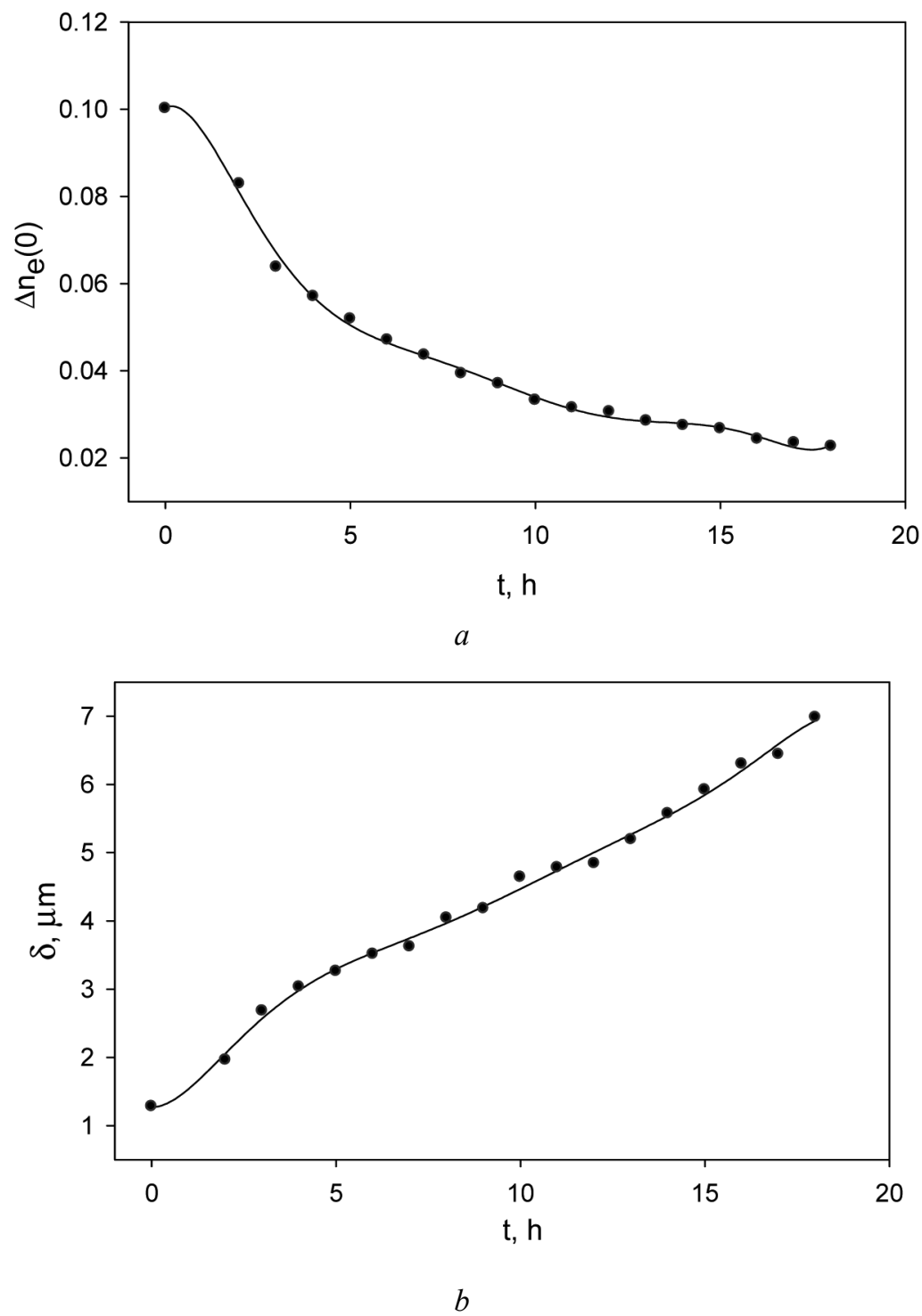

Рис. 2. Зависимость $\Delta n_{e}(0)(a)$ и глубины волноводного слоя $(b)$ от продолжительности отжига [Fig. 2. Dependence of $\Delta n_{e}(0)(a)$ and guide layer depth $(b)$ on annealing duration]

широких пределах, поэтому увеличение поглощения имеет место в широком диапазоне значений $v$. Асимметричный максимум поглощения, расщепляющийся в верхней части на два пика, обусловлен присутствием $\beta_{1}$-фазы и $\alpha$-фазы в протонообменных слоях. Декомпозиция данного пика на два пика с использованием функции Гаусса и метода Левенберга-Марквардта дает компоненты с параметрами, приведенными в табл. 1.

Пик со значением волнового числа $3481 \mathrm{~cm}^{-1}$, вероятно, соответствует $\alpha$-фазе, тогда как пик $3504 \mathrm{~cm}^{-1}$, возможно, обусловлен присутствием $\beta_{1}$-фазы в протонообменном слое. В то же время, в работе [11] для $\beta_{1}$-фазы приводится значение $v=3500 \mathrm{~cm}^{-1}$, а для $\alpha$-фазы $-v=3488 \mathrm{~cm}^{-1}$.
Для установления фаз, которые могут присутствовать в протонообменных слоях, проводилась также декомпозиция рентгеновских пиков; ее результаты представлены в табл. 2. Установление природы фаз проводилось путем сопоставления величин деформаций, полученных после декомпозиции пиков, со структурно-фазовой диаграммой.

Из табл. 2 следует, что после 1 ч отжига в протонообменных слоях присутствуют $\kappa_{2}$-фаза, $\kappa_{1}$-фаза и $\alpha$-фаза. Присутствие $\alpha$-фазы объясняется тем, что она в небольших количествах имеется даже в непротонированном ниобате лития [12]; кроме того, в результате диффузии в ходе протонного обмена и отжига некоторая часть протонов может оказаться 


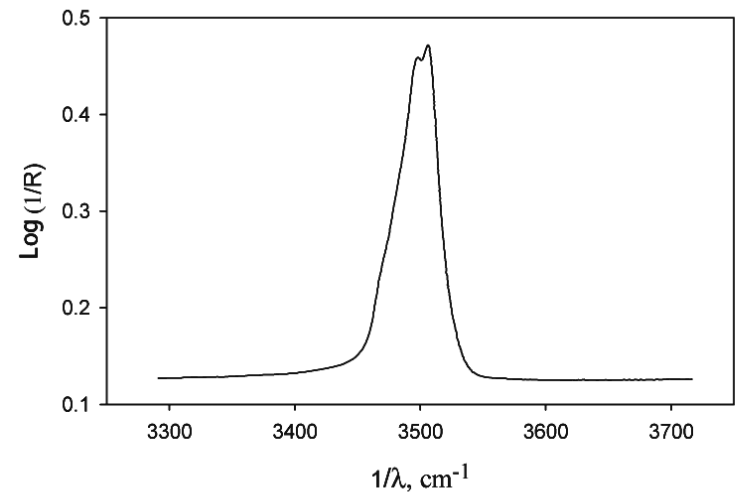

$a$

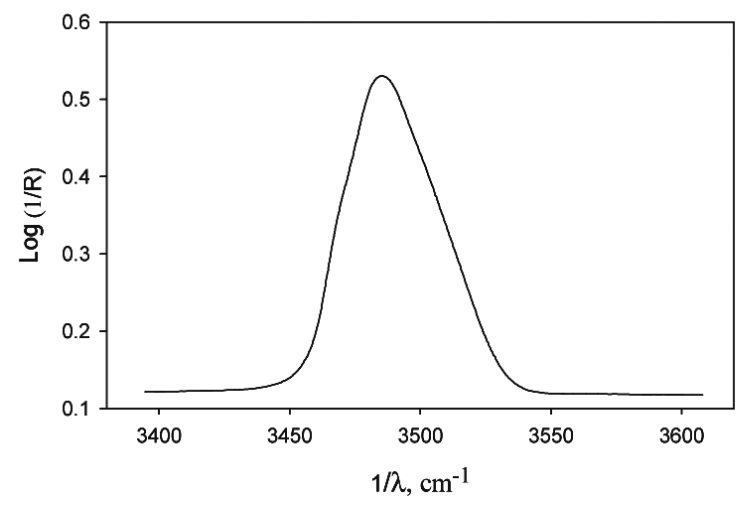

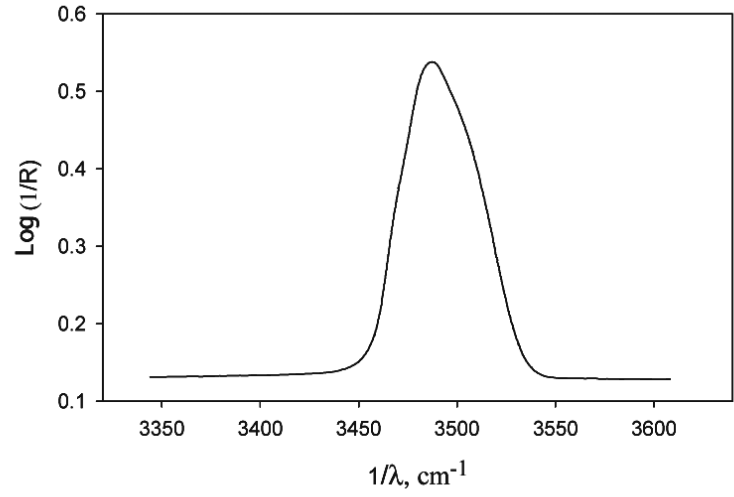

$b$

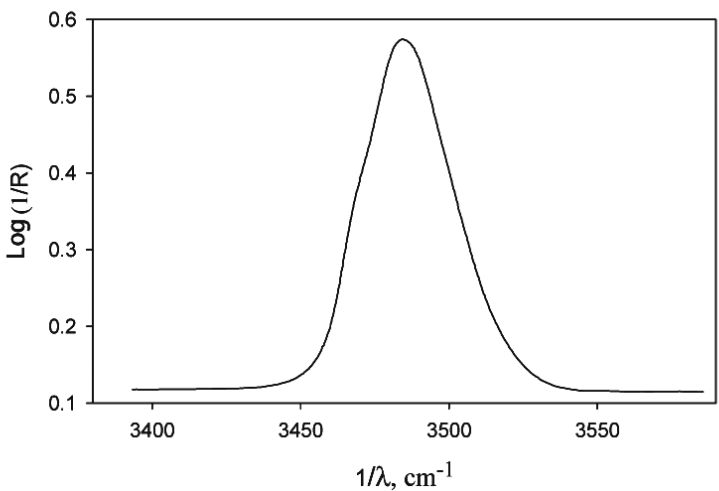

$d$

Рис. 3. ИК-спектры поглощения протонированного ниобата лития после отжига в течение, ч: $a-0, b-2, c-6, d-18$

[Fig. 3. IR absorption spectra of protonated lithium niobate after annealing of duration, h: $a-0, b-2, c-6, d-18$ ]

Таблица 1. Компоненты полосы поглощения при $\sim 3500 \mathrm{~cm}^{-1}$

[Table 1. Components of absorption band at $\sim 3500 \mathrm{~cm}^{-1}$ ]

\begin{tabular}{|c|c|c|}
\hline $\begin{array}{c}\text { Продолжительность отжига, ч } \\
\text { [Annealing duration, } \mathrm{h}]\end{array}$ & $\begin{array}{c}v_{\text {макс }}, \mathrm{cm}^{-1} \\
{\left[v_{\max }, \mathrm{cm}^{-1}\right]}\end{array}$ & $\begin{array}{c}\text { Относительная интенсивность } \\
\text { [Relative intensity] }\end{array}$ \\
\hline 0 & 3504 & 0.62 \\
& 3481 & 0.38 \\
\hline 2 & 3507 & 0.42 \\
& 3482 & 0.58 \\
\hline 4 & 3509 & 0.29 \\
& 3484 & 0.71 \\
\hline \multirow{2}{*}{6} & 3505 & 0.38 \\
\hline 15 & 3482 & 0.62 \\
\hline
\end{tabular}

за пределами слоев $\kappa_{2}$ - и к -фаз. Следует отметить, что микроскопические исследования с использованием поляризованного света в темном и светлом полях не выявили образования частиц к -фазы, как это имело место при аналогичном отжиге протонообменных слоев, сформированных на X-срезе кристалла $[8,9]$.
Декомпозиция второго порядка отражения дает сопоставимые величины деформаций и несколько большую долю фазы ниобата лития, поскольку в формировании отражения участвует поверхностный слой кристалла в два раза большей толщины (табл. 2). Следует отметить, что относительные интенсивности линий той или иной фазы, получен- 
Таблица 2. Деформации $\left(\varepsilon_{33}\right)$ и интегральные интенсивности линий $(I)$, полученные при декомпозиции отражений (006) и (0012)

[Table 2. Strains $\left(\varepsilon_{33}\right)$ and relative intensities $(I)$ of lines obtained at decomposing reflections (006) and (0012)]

\begin{tabular}{|c|c|c|c|c|c|c|c|}
\hline \multirow{2}{*}{ 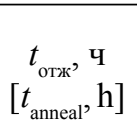 } & \multicolumn{3}{|c|}{$\begin{array}{c}\text { Деформация, } \varepsilon_{33} 10^{3} \\
{[\text { Strain }]}\end{array}$} & \multicolumn{4}{|c|}{$\begin{array}{c}\text { Относительная интенсивность, } I \\
\text { [Relative intensity] }\end{array}$} \\
\hline & $\kappa_{2}$ & $\kappa_{1}$ & $\alpha$ & $\kappa_{2}$ & $\kappa_{1}$ & $\alpha$ & $\mathrm{LiNbO}_{3}$ \\
\hline \multicolumn{8}{|c|}{$\begin{array}{l}\text { Отражение (006) } \\
\text { [Reflection (006)] }\end{array}$} \\
\hline 1 & 2.2 & 0.8 & 0.3 & 0.14 & 0.29 & 0.31 & 0.26 \\
\hline 2 & 1.6 & 1.0 & 0.4 & 0.06 & 0.24 & 0.25 & 0.45 \\
\hline 4 & - & 0.6 & - & - & 0.67 & - & 0.33 \\
\hline 6 & - & 0.7 & 0.9 & - & 0.64 & 0.01 & 0.35 \\
\hline 9 & - & 0.7 & 0.7 & - & 0.69 & 0.08 & 0.23 \\
\hline 12 & - & 1.0 & 0.6 & - & 0.01 & 0.81 & 0.18 \\
\hline 15 & - & - & 0.8 & - & - & 0.95 & 0.05 \\
\hline \multicolumn{8}{|c|}{$\begin{array}{l}\text { Отражение (0012) } \\
\text { [Reflection (0012)] }\end{array}$} \\
\hline 1 & 2.3 & 0.8 & 0.3 & 0.16 & 0.27 & 0.03 & 0.54 \\
\hline 2 & 1.6 & 0.7 & 0.3 & 0.14 & 0.23 & 0.08 & 0.55 \\
\hline 4 & - & 0.7 & 0.1 & - & 0.64 & 0.07 & 0.29 \\
\hline 6 & - & 1.0 & 0.4 & - & 0.54 & 0.24 & 0.21 \\
\hline
\end{tabular}

ные в результате декомпозиции экспериментальных пиков, использовались лишь для приближенной оценки долей соответствующих фаз.

Увеличение продолжительности отжига до 2 ч (рис. 1c) приводит к некоторому смещению пика $\kappa_{2}$-фазы, соответствующему снижению деформаций $\left(\varepsilon_{33}=1.6 \cdot 10^{-3}-\right.$ первый порядок отражения, $\varepsilon_{33}=2.0 \cdot 10^{-3}-$ второй порядок отражения) и доли $\kappa_{2}$-фазы (табл. 2). Асимметричность основного пика еще больше увеличивается (рис. 4). Деформации для к-фазы и $\alpha$-фазы несколько возрастают (табл. 2). Значение $\Delta n_{\mathrm{e}}$ на поверхности волновода указывает на присутствие $\kappa_{2}$-фазы в поверхностном слое после 2 ч отжига (рис. 2). Микроскопическое исследование не выявило образования частиц к -фазы в ходе дальнейшего отжига.

После отжига в течение 2 ч отсутствует ИК-поглощение, соответствующее $\beta_{2}$-фазе (рис. $3 b$ ), декомпозиция асимметричного пика $\left(v \sim 3500 \mathrm{~cm}^{-1}\right)$ дает близкие значения волновых чисел (табл. 1). Однако, в соответствии с результатами рентгеноструктурного анализа максимумы, полученные в результате декомпозиции, могут соответствовать $\kappa_{1}$ - и к зам $\left(v=3507 \mathrm{~cm}^{-1}\right)$ и $\alpha$-фазе $\left(v=3482 \mathrm{~cm}^{-1}\right)$.

При дальнейшем отжиге происходит монотонное снижение величины $\Delta n_{\text {е }}$ и увеличение глубины волновода. Характерной горизонтальной площадки, которая регистрировалась при отжиге протонообменных слоев на X-срезе и соответствовала переходу $\kappa_{2}$-фазы в $\kappa_{1}$-фазу с образованием частиц последней, на Z-срезе не наблюдалось.

После 4 ч отжига пик на дифрактограммах, соответствующий $\kappa_{2}$-фазе, исчезает, происходит дальнейшее увеличение асимметричности основного пика. Можно предположить, что после 4 ч отжига процесс фазового превращения кфазы в к-фазу (низкотемпературная) практически завершен. На это указывают и результаты модовой спектроскопии, поскольку величина $\Delta n$ е на поверхности волновода соответствует $\kappa_{1}$-фазе (рис. 2). Глубина волновода достигает 3 мкм. Это примерно соответствует толщине слоя на поверхности кристалла, от которого происходит отражение рентгеновских лучей при регистрации линии (006).

Декомпозиция основного пика указывает на присутствие $\kappa_{1}$-фазы со значением $\varepsilon_{33}=(0.6-0.7) \cdot 10^{-3}$

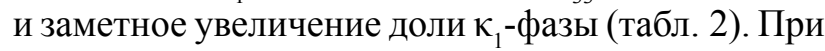
декомпозиции второго порядка отражения - линии (0012) - было выявлено лишь незначительное присутствие $\alpha$-фазы (доля $\alpha$-фазы - 0.07). Результаты оптической микроскопии здесь и в ходе дальнейшего отжига также не выявили образования частиц $\kappa_{1}$-фазы. Следовательно, в процессе отжига когерентность слоев, образованных из различных фаз, не нарушается.

Декомпозиция полосы поглощения (рис. 3c) дает два компонента с волновыми числами 3509 и 
$3484 \mathrm{~cm}^{-1}$ (табл. 1), которые могут соответствовать $\kappa_{1}$-фазе и $\alpha$-фазе.

После отжига в течение 6 и 9 ч регистрируется единственный асимметричный пик (рис. $1 d, e$ ), асимметричность данного пика максимальна при времени отжига 6 ч. Асимметричность пика вызвана присутствием $\kappa_{1}$ - и $\alpha$-фаз наряду с фазой ниобата лития (табл. 2). Величина деформаций, соответствующих $\kappa_{1}$-фазе, и ее содержание с увеличением продолжительности отжига (с 4 до 9 ч) изменяется незначительно, тогда как содержание $\alpha$-фазы постепенно увеличивается, одновременно снижается доля ниобата лития. Деформации, соответствующие $\alpha$-фазе, увеличиваются и при времени отжига 6 ч даже незначительно превышают деформации, характерные для $\kappa_{1}$-фазы, несмотря на меньшую концентрацию протонов в $\alpha$-фазе по сравнению с их концентрацией в $\kappa_{1}$-фазе. Такое соотношение деформаций не противоречит структурно-фазовой диаграмме [2]. Величина $\Delta n_{\mathrm{e}}(0)=0.037$ может соответствовать одновременному присутствию $\kappa_{1}$ - и $\alpha$-фаз.

Декомпозиция полосы поглощения (рис. 3c) после 6 ч отжига дает два компонента с волновыми числами 3505 и $3482 \mathrm{~cm}^{-1}$ (табл. 1), которые также могут соответствовать $\kappa_{1}$-фазе и $\alpha$-фазе. Снижение волнового числа первого компонента, вероятно, связано со снижением концентрации протонов в $\kappa_{1}$-фазе в результате отжига.

При времени отжига 12 ч существенно уменьшаются асимметричность пика и доля $\kappa_{1}$-фазы, значительно увеличивается доля $\alpha$-фазы (табл. 2). Вероятно, превращение $\kappa_{1}$-фазы в $\alpha$-фазу происходит, в основном, при отжиге за промежуток времени с 9 до 12 ч. Следует отметить, что асимметричность пика наблюдается в его правой части, что может быть обусловлено вкладом фазы ниобата лития. Такая же асимметричность наблюдается при времени отжига 15 ч (рис. 1f).

Некоторое увеличение деформаций $\kappa_{1}$-фазы (время отжига 12 ч), возможно, связано с тем, что оставшийся тонкий слой данной фазы располагается в непосредственной близости от поверхности кристалла. Именно в этом слое в ходе протонного обмена достигаются максимальные концентрации протонов и возникают наибольшие деформации. При времени отжига 15 ч в протонообменных слоях не обнаруживается $\kappa_{1}$-фаза. Протонообменный слой состоит преимущественно из $\alpha$-фазы (табл. 2). На это указывают и результаты ИК-спектроскопии, поскольку при временах отжига 15 и 18 ч имеется единственный пик поглощения при $3484 \mathrm{~cm}^{-1}$, соответствующий $\alpha$-фазе (рис. $3 d$ ).

\section{ВЫВОДЫ}

1. С использованием методов модовой спектроскопии, оптической микроскопии в поляризованном свете, рентгеноструктурного анализа и ИК-спектроскопии установлена последовательность фазовых превращений при отжиге (330 $\left.{ }^{\circ} \mathrm{C}\right)$ протонообменных слоев, сформированных на кристаллах ниобата лития (Z-срез): $\beta_{1}, \beta_{2}$-фазы $\rightarrow$ $\kappa_{2}$-фаза $\rightarrow \kappa_{1}$-фаза $\rightarrow \alpha$-фаза.

2. В процессе отжига в ходе фазовых превращений когерентность слоев, образованных из различных фаз, не нарушается, что обусловлено меньшей величиной деформаций (напряжений) формирующихся протонообменных фаз по сравнению с деформациями (напряжениями) на $\mathrm{X}$-срезе кристалла.

Работа выполнена при финансовой поддержке гранта РФФИ (проект № 17-43-590309 p_a).

\section{СПИСОК ЛИТЕРАТУРЫ}

1. Korkishko Yu. N., Fedorov V. A., Kostritskii S. M., Alkaev A. N., Maslennikov E. I., Paderin E. M., Apraksin D. V., Laurell F. // Microelectronic Engineering, 2003, vol. 69 , pp. 228-236.

2. Korkishko Yu. N., Fedorov V. A. // IEEE J. Sel. Top. Quantum Electron., 1996, vol. 2, № 2, pp. 187-196.

3. Kostritskii S. M., Korkishko Yu. N., Fedorov V. A., Sevostyanov O. G., Chirkova I. M., Mitrokhin V. P. // J. Appl. Spectroscopy, 2015, vol. 82, № 2, pp. 234-241.

4. Suchoski P. G., Findakly T. K., Leonberger F. J. // Optics Letters, 1988, vol. 13, № 11, pp. 1050-1052.

5. Korkishko Yu. N., Fedorov V. A. // Technical Physics, 1999, vol. 44, № 3, pp. 307-316.

6. Chen S., Baldi P., De Micheli M. P., Ostrowsky D. B., Leycuras A., Tartarini G., Bassi P. // J. Lightwave Technol., 1994, vol. 12, № 5, pp. 862-871.

7. Sun Jian, Xu Chang-qing // J. Appl. Phys., 2015, vol. 117, article ID 043102, 8 pp.

8. Mushinsky S. S., Kichigin V. I., Petukhov I. V., Permyakova M. A., Shevtsov D. I. // Ferroelectrics, 2017, vol. 508, № 1, pp. 40-48.

9. Мушинский С. С., Петухов И. В., Пермякова М. А., Кичигин В. И., Малинина Л. Н., Волынцев А. Б. // Конденсированные среды и межфазные гранищы, 2017, т. 19, № 4, с. 551-560.

10. Колосовский Е. А., Петров Д. В., Царев А. В. // Ж. квант. электрон., 1981, т. 8, № 12, с. 2557-2568.

11. Kuneva M., Tonchev S., Thatsi E., Lampakis D. // J. Optoelectronics and Advanced Materials, 2005, vol. 7, № 1 , pp. 549-552.

12. Cabrera J. M., Olivares J., Carrascosa M., Rams J., Müller R., Diéguez E. // Advances in Physics, 1996, vol. 45, № 5, pp. 349-392. 


\title{
PECULIARITIES OF STRUCTURAL PHASE TRANSFORMATIONS IN PROTON EXCHANGED LAYERS OF Z-CUT LITHIUM NIOBATE CRYSTAL DURING ANNEALING
}

\author{
(C) 2018 I. V. Petukhov, S. S. Mushinsky, M. A. Permyakova, V. I. Kichigin, \\ L. N. Malinina, A. B. Volyntsev \\ Perm State University, 15 Bukirev str., Perm, Russia \\ e-mail:Petukhov-309@yandex.ru
}

Received 17.05.2018

\begin{abstract}
Lithium niobate crystals are widely used in the manufacturing of integrated optical phase and intensity modulators. The production method is based on the formation of optical waveguides in the surface layers of $\mathrm{LiNbO}_{3}$ by means of proton exchange. Subsequent annealing of proton-exchanged structures is usually performed in order to obtain stable and low-loss waveguides.

The aim of this paper is to study phase transitions in the surface layer of proton-exchanged waveguides on Z-cuts of lithium niobate crystals during annealing. In our research, the following methods were used: polarized light optical microscopy (bright field and dark field), mode spectroscopy (determination of the refractive index profile), IR-spectroscopy, and XRD ( $\theta / 2 \theta$ curves). Congruent lithium niobate (Sipat) was used as a material. Proton exchange was carried out in molten benzoic acid at $190{ }^{\circ} \mathrm{C}$ for 2 hours. The samples were then annealed at 1 -hour intervals at $330^{\circ} \mathrm{C}$. The total duration of annealing was 18 hours.

The sequence of the phase transformations at annealing proton-exchange layers on Z-cut lithium niobate crystals is similar to that on $\mathrm{X}$ cut: $\beta_{1}, \beta_{2}$-phase $\rightarrow \kappa_{2}$-phase $\rightarrow \kappa_{1}$-phase $\rightarrow \alpha$-phase. The difference is that $\kappa_{1}$-phase particles are not formed during annealing and the coherence of the layers of different phases is not disturbed. This results from a lesser magnitude of strains in proton-exchange phases on $\mathrm{Z}$ cut $\mathrm{LiNbO}_{3}$ as compared to $\mathrm{X}$ cut.
\end{abstract}

Keywords: lithium niobate, Z-cut, proton exchange, annealing, phase transformation.

DOI: https://doi.org/10.17308/kcmf.2018.20/581

\section{ACKNOWLEDGEMENTS}

This work was supported by grant Russian Foundation for Basic Research No. 17-43-590309 p_a.

\section{REFERENCES}

1. Korkishko Yu. N., Fedorov V. A., Kostritskii S. M., Alkaev A. N., Maslennikov E. I., Paderin E. M., Apraksin D. V., Laurell F. Microelectronic Engineering, 2003, vol. 69, pp. 228-236. DOI: 10.1016/S0167-9317(03)00302-2

2. Korkishko Yu. N., Fedorov V. A. IEEE J. Sel. Top. Quantum Electron., 1996, vol. 2, no. 2, pp. 187-196. DOI: 10.1109/2944.577359

3. Kostritskii S. M., Korkishko Yu. N., Fedorov V. A., Sevostyanov O. G., Chirkova I. M., Mitrokhin V. P. J. Appl. Spectroscopy, 2015, vol. 82, no. 2, pp. 234-241. DOI: 10.1007/s10812-015-0091-2

4. Suchoski P. G., Findakly T. K., Leonberger F. J. Optics Letters, 1988, vol. 13, no. 11, pp. 1050-1052. DOI: 10.1364/OL.13.001050

5. Korkishko Yu. N., Fedorov V. A. Technical Physics, 1999, vol. 44, no. 3, pp. 307-316. DOI: 10.1134/1.1259243
6. Chen S., Baldi P., De Micheli M. P., Ostrowsky D. B., Leycuras A., Tartarini G., Bassi P. J. Lightwave Technol., 1994, vol. 12, no. 5, pp. 862-871. DOI: 10.1109/50.293979

7. Sun Jian, Xu Chang-qing. J. Appl. Phys., 2015, vol. 117, article ID 043102, 8 pp. DOI: $10.1063 / 1.4906222$

8. Mushinsky S. S., Kichigin V. I., Petukhov I. V., Permyakova M.A., Shevtsov D. I. Ferroelectrics, 2017, vol. 508, no. 1, pp. 40-48. DOI: 10.1080/00150193.2017.1286702

9. Mushinsky S. S., Petukhov I. V., Permyakova M. A., Kichigin V. I., Malinina L. N., Volyntsev A. B. Condensed Matter and Interphases, 2017, vol. 19, no. 4, pp. 551-560. Available at: http://www.kcmf.vsu.ru/resources/ t_19_4 2017_011.pdf (in Russ.)

10. Kolosovskii E. A., Petrov D. V., Tsarev A. V. Sov. J. Quantum Electron., 1981, vol. 11, no. 12, pp. 1560-1566. DOI: 10.1070/ QE1981v011n12ABEH008650

11. Kuneva M., Tonchev S., Thatsi E., Lampakis D. J. Optoelectronics and Advanced Materials, 2005, vol. 7, no. 1, pp. 549-552. Available at: http://joam.inoe.ro/arhiva/pdf7 1/Kuneva.pdf

12. Cabrera J. M., Olivares J., Carrascosa M., Rams J., Müller R., Diéguez E. Advances in Physics, 1996, vol. 45, no. 5, pp. 349-392. DOI: 10.1080/00018739600101517 
Петухов Игорь Валентинович - к. х. н., доцент кафедры физической химии, Пермский государственный национальный исследовательский университет; тел.: +7(342) 2396789, e-mail: petukhov309@yandex.ru

Мушинский Сергей Сергеевич - инженер, Пермский государственный национальный исследовательский университет; тел.: +7(342) 2396789, еmail: sergey.mushinsky@gmail.com

Пермякова Мария Александровна - магистрант кафедры физической химии, Пермский государственный национальный исследовательский университет; тел.: +7(342) 2396789

Кичигин Владимир Иванович - с. н. с. кафедры физической химии, Пермский государственный национальный исследовательский университет; тел.: +7(342) 2396452, e-mail: kichigin@psu.ru

Малинина Людмила Николаевна - инженер кафедры физики твердого тела, Пермский государственный национальный исследовательский университет; тел.: +7(342) 2396383

Вольнщев Анатолий Борисович - д. ф.-м. н., профессор, заведующий кафедрой физики твердого тела, Пермский государственный национальный исследовательский университет; тел.: +7(342) 2396410, e-mail: voland@psu.ru
Igor V. Petukhov - Cand. Sci. (Chem.), Associate Professor, Department of Physical Chemistry; Perm State University, tel.: +7(342) 2396789, e-mail: petukhov-309@yandex.ru

Sergey S. Myshinsky - Engineer, Perm State University; tel.: +7(342) 2396789, e-mail: sergey.mushinsky@gmail.com

Mariya A. Permyakova - graduate student, Department of Physical Chemistry, Perm State University; tel.: +7(342) 2396789

Vladimir I. Kichigin - Cand. Sci. (Chem.), Senior Researcher, Department of Physical Chemistry, Perm State University; tel.: +7(342) 2396452, e-mail: kichigin@psu.ru

Ljudmila N. Malinina - Engineer of Solid State Physics Department, Perm State University; tel.: $+7(342) 2396383$

Anatoliy B. Volyntsev - Dr. Sci. (Phys.-Math.), Professor, Head of Solid State Physics Department, Perm State University; tel.: +7(342) 2396410, e-mail: voland@psu.ru 